\title{
Factores determinantes del nivel de compromiso medioambiental voluntario adquirido por los hoteles cotizados europeos
}

Patricia Milanés

Montero

Universidad de Extremadura pmilanes@unex.es

\section{Esteban Pérez}

\section{Calderón}

Universidad de Extremadura estperez@unex.es

\section{Francisco Javier}

Ortega Rossell

Universidad de Extremadura jortega@unex.es

\section{Resumen}

Ante las importantes presiones que la actividad hotelera ejerce sobre el medioambiente, la ausencia de regulación de las actuaciones responsables y la escasez de investigaciones al respecto aparece la imperiosa necesidad de aportar luz en este sentido. Por ello, en este trabajo se analiza el compromiso medioambiental corporativo de los hoteles cotizados europeos; se utiliza como metodología el análisis de contenidos y se detectan los factores determinantes de las actuaciones responsables con el medioambiente con el propósito de contribuir a la construcción de un marco de referencia objetivo de medida de los avances en sostenibilidad de la actividad turística. Además, se contribuye al "argumento comercial" analizando la relación existente entre la rentabilidad empresarial y el desempeño medioambiental. Los resultados muestran que la mayoría de los hoteles analizados carece de política de protección medioambiental; asimismo, el tamaño, la normativa contable adoptada y la pertenencia a grupos son factores determinantes de la adopción de una postura más o menos responsable. Por otro lado, la rentabilidad empresarial resulta ser una de las ventajas de la integración de las cuestiones medioambientales en la estrategia corporativa de las empresas.

Palabras clave: compromiso medioambiental, factores determinantes, sector hotelero. 


\title{
Determinants of voluntary environmental commitment acquired by Euro- pean listed hotels
}

\begin{abstract}
Due to the significant pressures that hotels exert on the environment and the lack of regulation and research about it, it is necessary to provide solutions for this matter as soon as possible. That is the reason why this paper analyzes corporate environmental commitment of European listed hotels, with the use of a content analysis methodology and by identifying the determinants of environmentally responsible actions. The purpose is to contribute to building a framework to measure the advances in sustainable tourism. Furthermore, it contributes to the "commercial argument" by means of the study of the relationship between return on investment and environmental performance. The results show that most of the hotels analyzed do not have an environmental protection policy and that the size, accounting practice and type of financial information disclosure (consolidated or unconsolidated data), influence that commitment. In addition, a higher return on investment is one of the advantages of voluntary environmental commitment.
\end{abstract}

Keywords: environmental commitment, determinants, hotel industry.

\section{Introducción}

Las importantes presiones que la actividad turística ejerce sobre el medio ambiente (generación de residuos urbanos, aumento del consumo de agua y otros residuos, vertidos de aguas residuales en caso de instalaciones de depuración insuficientes), agravadas por la estacionalidad y su concentración espacial, están centrando los esfuerzos de la Comisión Europea en el fomento de un turismo asentado sobre las bases de la competitividad y la sostenibilidad medioambiental, social y económica, en consonancia con la propuesta de la Global Reporting Initiative (GRI) basada en el enfoque del "triple bottom line" económico, social y medioambiental.

Si bien se han adoptado buenas prácticas en responsabilidad social en algunos países europeos como Bélgica, Dinamarca, Alemania, Irlanda, Holanda, Austria, Reino Unido, entre otros (García de Cortázar, 2008), no existen reglas objetivas al respecto. Además, tal como apunta Bien (2006: 19), todos los sistemas de certificación de turismo como Portal DestiNet, Asociación VISIT (Iniciativas Voluntarias para la Sostenibilidad en Turismo), European Flower — en el nivel europeo- y Criterios de la Organización Mundial del Turismo (OMT) —en el nivel internacio- 
nal - son voluntarios; es decir, no se requiere que ninguna empresa sea certificada, ni puede imponerse ningún sistema de certificación específico a las empresas que deseen obtener la certificación.

En cuanto a las orientaciones existentes en la actualidad para aquellas empresas que deseen guiar sus actuaciones responsables con el medio ambiente, cabe citar, aparte de los criterios de turismo sostenibles de la OMT, las siguientes:

- El modelo de gestión medioambiental regulado por la Comisión Europea, a través del Reglamento (CEE) No 1836/93 (EMAS), el cual permite que las empresas del sector industrial se adhieran con carácter voluntario a un sistema comunitario de gestión y auditoría medioambientales; dicho sistema prácticamente coincide con el incluido en la norma ISO 14001: 1996(Sistemas de Gestión Medioambiental), reconocida en el año 1997 por la Comisión Europea de conformidad con el artículo 12 del Reglamento. Actualmente, el Reglamento que regula el EMAS es el 761/2001, del 19 de marzo de 2001, que recoge los puntos básicos del Reglamento 1836/93, pero amplía su cobertura en aras de conseguir una mayor adhesión empresarial. En el sector hotelero, en concreto, los hoteles que tienen implantado un SGMA de acuerdo con las normas 14001 o EMAS utilizan los indicadores específicos de comportamiento ambiental (operacionales) que están relacionados con la "eficiencia" en el uso de los recursos (consumo de recursos / servicios prestados o capacidad del hotel, etc.) (Burgos-Jiménez, 2001).

- La guía para la elaboración de memorias de sostenibilidad elaborada por La Global Reporting Initiative (GRI), la cual — según Moneva et al. (2006)— representa la institución más relevante en la actualidad en el ámbito de la comunicación corporativa sobre desarrollo sostenible. En ella se presentan una serie de indicadores: principales y adicionales. Los primeros se refieren a los aspectos materiales, energía, agua, biodiversidad, emisiones, vertidos y residuos, productos y servicios, así como cumplimiento normativo.

No obstante, ante la inexistencia de normas objetivas que regulen las actuaciones responsables con el medioambiente, se hace necesario contar con un marco de referencia objetivo para medir los avances que se van produciendo en la sostenibilidad de la actividad turística (Garrod y Fyall, 1998). De este modo se evitaría la coexistencia de diferentes interpretaciones en función de intereses particulares, 
que en la actualidad están provocando conflictos en lugar de potenciar los avances en relación con la sostenibilidad de la actividad turística (McKercher, 1993). En esta misma línea, la OMT — que ha venido desarrollando desde 2007 un conjunto de criterios e indicadores de turismo sostenible para el uso por parte de los hoteles y operadores de turismo, principalmente - advierte la ventaja de utilizarlos como referencia en todo el mundo, con las adaptaciones derivadas de las diferencias regionales y sectoriales para posibilitar la comparación del rendimiento entre las empresas y las regiones.

En cuanto a las aportaciones de las investigaciones realizadas en este campo, concretamente en la industria hotelera, cabe señalar que - a pesar de que se ha constatado la importancia de los daños causados en el medio ambiente, dado la naturaleza de sus funciones, características y servicios, consumo de cantidades sustanciales de energía, agua y productos perecederos (Mensah, 2004; Trung y Kumar, 2005; Erdogan y Baris, 2006) — son muy escasos los estudios existentes sobre el compromiso medioambiental de las empresas turísticas; los que se han llevado a cabo se centran exclusivamente en hoteles de tres, cuatro y cinco estrellas (Erdogan y Baris, 2006; Blanco et al., 2008). Además, existe una reciente demanda de trabajos de investigación en relación con la sostenibilidad de la actividad turística en Europa y en todo el mundo (Chung y Parker, 2009; Hopwood, 2009; Blanco et al., 2008).

Por todo ello, el presente trabajo persigue determinar el nivel de compromiso medioambiental adquirido de forma voluntaria por las principales cadenas hoteleras cotizadas europeas a partir de la información contenida en sus estados financieros y analizar en qué medida dicho compromiso puede ser fuente de ventaja competitiva para el negocio ("argumento comercial"). Además, se ofrece evidencia empírica acerca de los factores determinantes de la responsabilidad medioambiental adoptada con la finalidad de complementar las aportaciones de la literatura previa en este sentido. Husillos Carqués (2007) defiende la relevancia de investigar a este respecto debido a la dificultad de llegar a conclusiones fiables sobre el comportamiento medioambiental de las empresas analizando la información que emiten en sus estados financieros (Larrinaga et al., 2002; Llena et al., 2007). 


\section{Revisión de la literatura}

\section{Compromiso medioambiental voluntario}

La mayoría de las investigaciones sobre responsabilidad medioambiental mide la variable compromiso con el medioambiente a partir de la información obtenida a través de entrevistas en profundidad o encuestas de opinión. Tal es el caso de García Rodríguez y Armas Cruz (2007), quienes para definir la variable responsabilidad social y medioambiental (RSM) realizan entrevistas en profundidad a los gerentes de 15 hoteles españoles; como definición obtienen que la empresa socialmente responsable es la que cuenta con políticas y lleva a cabo estrategias de protección del medio ambiente (reciclaje de residuos, ahorro de recursos y energía, prevención de la contaminación, etc.); además, la empresa se comporta de forma ética y participa en la solución de los problemas sociales.

Según Tarí et al. (2009), entre las variables medioambientales más utilizadas se encuentran: a) variables relativas a la gestión medioambiental: prácticas, iniciativas, tecnologías, sistemas de gestión medioambiental (Judge y Douglas, 1998; Klassen y Whybark, 1999); b) variables de desempeño ambiental: en sentido positivo (reducción de emisiones) y negativo (generación de emisiones) (Hart y Ahuja, 1996; Wagner et al., 2002). Los autores utilizan, en una encuesta de opinión, la escala propuesta por Carmona-Moreno et al. (2004) para medir el compromiso medioambiental, la cual incluye doce prácticas medioambientales divididas en dos apartados: 1) compromiso medioambiental básico (compra de productos ecológicos, facilitar al cliente la colaboración con el medioambiente, reducción del uso de productos ambientalmente peligrosos, prácticas de ahorro de energía, prácticas de ahorro de agua y recogida selectiva de residuos sólidos) y 2) compromiso medioambiental avanzado (la empresa capacita a sus empleados en cuestiones ambientales, se compensa a los empleados por sus iniciativas medioambientales, se usan argumentos ecológicos en las campañas de marketing, organización de actividades ambientales por parte de la empresa, la empresa adopta un enfoque de compromiso medioambiental a largo plazo y la cuantificación del ahorro de los costos ambientales). Asimismo, estos autores distinguen un grupo de hoteles españoles de tres, cuatro y cinco estrellas con una gestión medioambiental proactiva y otro grupo, más pequeño, con una gestión medioambiental reactiva.

Llena Macarulla (1999) define el indicador de gestión medioambiental (IGMA) como la suma, para cada empresa encuestada, de los valores correspondientes a 
las siguientes variables: incorporación de un sistema o política medioambiental; existencia de responsable medioambiental específico; existencia de documento escrito de la gestión o política; existencia de certificación medioambiental; existencia de departamento medioambiental; participación del departamento en la toma de decisiones; realización de actividades de naturaleza preventiva; realización de auditorías internas y externas; elaboración de informes específicos para la gestión medioambiental.

En cuanto a los comportamientos empresariales encontrados por las investigaciones empíricas en relación con la asunción de un compromiso medioambiental por parte de las empresas turísticas, cabe señalar que en la mayoría de ellas se ha detectado la existencia de distintos niveles de compromiso.

Así, Erdogan y Baris (2007) realizan un estudio que concluye que las políticas y prácticas de los hoteles del Ankara generalmente carecen de atributos relevantes de protección y conservación medioambiental y que la mayoría de los gerentes de los hoteles carecen del conocimiento y el interés medioambiental necesarios para identificar los objetivos básicos de la responsabilidad social y medioambiental.

En Blanco et al. (2009) se distinguen dos tipos de iniciativas medioambientales voluntarias por parte de las empresas turísticas: a) inclusión de consideraciones ambientales en su gestión operativa, que puede afectar directamente al rendimiento económico a través de ventajas competitivas y financieras (el uso más eficiente de las materias primas, la reducción de la contaminación, compra más verde, etc.); b) realización de inversiones medioambientales para mejorar directamente la dimensión de los resultados del medioambiente. Estos autores consideran tanto los compromisos unilaterales como los acuerdos negociados y los programas de certificación voluntaria (Khanna, 2001; Delmas y Keller, 2005) como iniciativas voluntarias de protección medioambiental porque cumplen dos características básicas: 1) los promotores de las iniciativas no están obligados por ley a poner en marcha el sistema y 2) los grupos destinatarios no están obligados a aplicarlas (OMT, 2002).

Centrándonos en la industria hotelera europea, Erdogan y Barris (2006) advierten que en los países de la Unión parece existir una mayor concientización con el medio ambiente, ya que más de la mitad (56.9\%) de los gerentes de los hoteles de Suiza han incluido en su plan de negocio alguna declaración de política medioambiental, frente al 4\% en Polonia (Bohdanowicz, 2005). Además, un estudio coor- 
dinado por PricewaterhouseCoopers en los hoteles europeos concluye que el $80 \%$ posee una política medioambiental (Clark y Siddall, 2001); en 2004, esta misma consultora vuelve a obtener un balance positivo al respecto, aunque sólo analiza los catorce mayores hoteles europeos que cotizan en bolsa.

Influencia del compromiso medioambiental en la rentabilidad empresarial y factores determinantes del nivel de responsabilidad adquirido

Entre las ventajas que la literatura atribuye a la integración de las cuestiones medioambientales en la estrategia corporativa de las empresas (García Rodríguez et al., 2006) se encuentran mejoras en la eficiencia, aumento de la calidad de los productos, incrementos en la cuota de mercado, acceso a nuevos mercados, satisfacción y motivación de los empleados, mejoras en las relaciones con la comunidad, acceso a ayuda financiera, entre otros (Beaumont et al., 1993, citado en García Rodríguez y Armas Cruz, 2006; Guimaraies y Liska, 1995; Porter y Van der Linde, 1995; Shrivastava, 1995; entre otros). Sin embargo, uno de los grandes desafíos de la responsabilidad social empresarial en Europa es la falta de información sobre la relación que existe entre la responsabilidad social y los resultados económicos de las empresas, es decir, "el argumento comercial" (García de Cortázar, 2008).

Ciertamente, la literatura previa sobre las consecuencias económicas de las acciones medioambientales voluntarias está basada, fundamentalmente, en el análisis de la industria manufacturera. En este sentido, Blanco et al. (2008) desarrollan un importante estudio sobre los incentivos económicos, publicado en la prestigiosa revista Tourism Management, los que conducen a las empresas turísticas a desarrollar actuaciones medioambientales de forma voluntaria; en dicho estudio concluyen que ante la inexistencia de estudios empíricos sobre el compromiso medioambiental de las empresas turísticas se hace necesario investigar en esta línea aprovechando los esfuerzos realizados por la OMT para ofrecer indicadores de sostenibilidad para la empresa turística. Además, tal como constatan García Rodríguez y Armas Cruz (2006), no existe un posicionamiento claro en cuanto a la relación existente entre compromiso medioambiental y performance, lo cual puede deberse, según De Burgos y Céspedes (2001) y Worrell et al. (1995), al uso de diferentes muestras de empresas, especialmente de empresas de diferentes sectores industriales; al uso de indicadores distintos en cada trabajo para medir las variables protección medioambiental y performance; a la inclusión o no en los diferentes modelos estimados de algunos aspectos de los ámbitos interno y externo de la organización que, directa o indirectamente, influyen en la protección del 
medio ambiente y el rendimiento económico; o al horizonte temporal para el que se analiza dicha relación (corto o largo plazo).

Entre las investigaciones que relacionan compromiso medioambiental con rentabilidad empresarial coexisten posturas a favor y en contra de la existencia de una relación positiva. Hart (1995), Russo y Fouts (1997), así como Sharma y Vredenburg (1998) coinciden en que las medidas que puedan ser desarrolladas por una empresa para la protección medioambiental pueden también permitirle obtener ventajas competitivas sostenibles. Sin embargo, en García Rodríguez y Armas Cruz (2006) se realiza una amplia revisión de la literatura sobre la existencia de una relación, positiva o negativa, entre responsabilidad medioambiental adquirida por la empresa y performance; asimismo, comprueban que existen estudios que demuestran la existencia de una relación negativa (Walley y Whitehead, 1994) y estudios que demuestran una relación positiva (Porter y Vander Linde, 1995). Según Blanco et al. (2008) no existen consecuencias económicas comunes para todos los tipos de iniciativa de gestión medioambiental (Hart y Ahuja, 1996; Judge y Douglas, 1998; King y Lenox, 2002; Klassen y Whybark, 1999; Rennings et al., 2006; Russo y Fouts, 1997; Thomas, 2001). Por su parte, Ambec y Lanoie (2007), lejos de pretender mostrar que una reducción de la contaminación está siempre acompañada de un mejor desempeño financiero, argumentan que los gastos realizados para reducir la contaminación pueden ser parcial o totalmente compensados, en algún momento, con ganancias obtenidas por otro lado; de hecho, estos autores muestran - a través de un estudio sistemático de todas las actuaciones ambientales que pueden contribuir a un incremento de ingresos- que ese tipo de ganancias pueden conducir a un mejor desempeño ambiental y financiero.

García Rodríguez y Armas Cruz (2007) realizan un estudio empírico centrado en el sector hotelero español con el que concluyen que existe una relación fuerte y positiva entre la responsabilidad social ambiental adquirida por la firma y su performance. Sin embargo, en Blanco et al. (2008) se analizan los resultados de un estudio desarrollado en el nivel europeo por Kassinis y Soteriou (2003) en el que no se llega a esta misma conclusión; los autores aplican un modelo de ecuaciones estructurales a una muestra de hoteles "superior deluxe" de los destinos turísticos de Europa con más de 10 millones de turistas por año (Austria, Francia, Alemania, Grecia, Italia, Portugal, España y Reino Unido) y justifican la existencia de una influencia significativa de las prácticas de gestión medioambiental en la satisfacción y de la satisfacción en los resultados económicos, pero no encuentran una relación directa entre prácticas de gestión medioambiental y resultados. Esto significa que 
cualquier influencia de las prácticas de gestión medioambiental en los resultados económicos de los hoteles se canaliza a través de un efecto de la demanda y no a través de la reducción de costos, tal como apoyan los estudios de Rivera (2004) y Parra et al. (2004).

En esta misma línea, Tarí et al. (2009), tras analizar los estudios previos sobre la relación existente entre gestión medioambiental y performance, concluyen que existen relaciones mixtas. Aunque la mayoría de los estudios demuestran los efectos positivos de la gestión medioambiental en el performance de la empresa, estos autores lo miden, considerado en términos de resultados operativos y financieros (Venkatraman y Ramanujam, 1986), a través de tres variables: ratio de ocupación por habitación, beneficio bruto de explotación (GOP) y beneficio bruto de explotación por habitación disponible por día (GOPPAR per day). Mediante un ANOVA y un análisis de regresión demuestran la relación existente entre los tres niveles de compromiso con la calidad y la protección medioambiental detectados y el performance. Lo anterior también se efectuó en una muestra de 301 hoteles españoles de tres, cuatro y cinco estrellas.

En cuanto a la literatura existente en relación con los factores determinantes del nivel de responsabilidad medioambiental adquirido por las empresas turísticas, que es otro de los objetivos planteados en este trabajo para el caso de los hoteles cotizados europeos, Husillos Carqués (2007) defiende la relevancia de investigar los motivos que orillan a las empresas a informar sobre su impacto medioambiental, dado la dificultad de llegar a conclusiones fiables sobre el comportamiento medioambiental de las firmas españolas analizando la información que emiten en sus estados financieros (Larrinaga et al., 2002; Llena et al., 2007).

Se observa una tendencia generalizada a emplear un ANOVA para analizar los factores determinantes del nivel de responsabilidad medioambiental adquirido voluntariamente por las empresas turísticas: influencia de los stakeholders, tamaño (Carmona Moreno et al., 2004) precio de la habitación (Rivera, 2002; Huybers y Bennett, 2002; Claver-Cortés et al., 2007).

Llena Macarulla (1999) contrasta el nivel de compromiso medioambiental de la empresa para una serie de factores por considerar que pueden ser diferenciadores de comportamientos. Se trata del tamaño de la entidad, de la nacionalidad de la matriz, del sector y de la política de gestión medioambiental (tener o no implantado un Sistema de Gestión Medioambiental, SGMA). En esta misma línea, 
Tarí et al. (2009) identifican distintas agrupaciones de hoteles españoles según su compromiso con la gestión medioambiental; los describen utilizando como variables características de la industria hotelera el número de estrellas, el tamaño, las instalaciones de hotel disponibles, la afiliación de la cadena y el precio de la habitación. Encuentran que el compromiso con la gestión medioambiental de su actividad incrementa cuando lo hacen todas estas variables, excepto la afiliación de la cadena.

\section{Diseño de la investigación. Población, metodología, variables y pruebas esta- dísticas}

Dada la escasez de estudios europeos sobre el nivel de compromiso medioambiental en el sector hotelero, nuestro análisis se centra en la población de hoteles cotizados pertenecientes a la Unión Europea que se encuentran registrados en la base de datos AMADEUS a la fecha de realización de este trabajo, es decir, N=69. Para evitar los posibles efectos de la crisis sobre la situación patrimonial y los resultados de estas firmas, e incluso sobre sus actuaciones medioambientales, se han tomado todos los hoteles activos en el año 2007 (Ver Anexo 1), tanto pertenecientes a grupos que presentan sus cuentas consolidadas como aquellos que no forman parte de grupos y presentan la información financiera individual.

La mayoría de los estudios existentes al respecto utilizan un cuestionario para la obtención de datos secundarios (Erdogan, Emin Baris, 2007). Sin embargo, la metodología de análisis empleada en este trabajo ha sido el análisis de contenidos, que consiste en clasificar la información divulgada en los informes anuales en varios grupos de elementos que recogen los aspectos medioambientales que se quieren analizar (Archel y Lizarraga, 2001; Husillos Carqués, 2007; Krippendorf, 1990); se asume la existencia de una tendencia generalizada por parte de las empresas a divulgar información sobre sus actuaciones responsables con el medio ambiente, tal como defiende la teoría de la legitimidad (Llull Gilet, 2004; FEE, 1993-1996; Giner Inchausti, 1992; Moneva y Llena, 1996; Carrasco y Larrinaga, 1995; Peyró Vilaplana, 1997; Ripoll y Crespo, 1998; Archel y Lizarraga, 2000; Harte y Owen, 1991; Roberts, 1991; Niskala y Pretes, 1995; Deegan y Rankin, 1996; De Beelde y Willequet, 2000).

Concretamente, se analiza el contenido de toda la información suministrada en las web corporativas (Adam y Frost, 2006) de los hoteles integrantes de la población; esto es, las cuentas anuales, el informe de gestión, el informe anual, la carta del 
presidente (Archel y Lizarraga, 2001) y los informes de sostenibilidad o gobierno corporativo en su caso (Aranguren y Ochoa 2008; Buhr y Freedman, 1996; Hibbitt, 2003).

En cuanto a las variables empleadas para medir el compromiso medioambiental adquirido por los mayores hoteles cotizados pertenecientes a la Unión Europea a partir de la información suministrada al respecto en sus páginas web, éstas han sido diseñadas a partir de la literatura previa y los criterios de turismo sostenible de la OMT y se organizan en dos bloques.

En el primer bloque, dedicado a los aspectos de asunción de compromiso medioambiental, se incluyen las siguientes variables, expresadas en términos dicotómicos, que toman el valor 1 cuando aparece el aspecto en la información contable y el valor 2 cuando no aparece:

a) Variables relativas al nivel de compromiso medioambiental asumido (Erdogan y Baris, 2006; Lull, 2004; Moneva, 2006; Reglamento CEE 1836/93 (EMAS); Global Reporting Initiative (GRI), 2000): A1. Hotel no comprometido con el medio ambiente; A2. Hotel con política o programa medioambiental; A3. Hotel que elabora un informe de evaluación del impacto ambiental; A4. Hotel certificado con ISO 14000. Premios y galardones; A5. Hotel con otras certificaciones: Flor Europea, ASOC VISIT, etc.; A6. Hotel con un Sistema de Gestión Medioambiental tipo EMAS, elaboración de memoria medioambiental, verificación. En relación con los Criterios GRI cumplidos en la memoria de sostenibilidad, se incluyen las variables: $A 61$. Aspecto: Materiales; A62. Aspecto: Energía; A63. Aspecto: Agua; A64. Aspecto: Biodiversidad; A65. Aspecto: Emisiones, vertidos, residuos; A66. Aspecto: Productos y servicios; A67. Aspecto: Cumplimiento normativo; A68. Nivel de aplicación de criterios GRI.

b) Variables relativas al tipo de actuación medioambiental emprendida: Siguiendo a Blanco et al. (2009), en este apartado se incluyen: B1. Variables representativas de actuaciones medioambientales que afectan a su rendimiento económico (B11. Política de compras favorecedora de productos ambientalmente amigables (compra verde); B12. Uso más eficiente de materias primas; B13.Adopción de medidas para reducir el consumo total de energía y fomento del uso de la energía renovable; B14. Disminución de la contaminación del aire y del suelo y la causada por 
el ruido, la iluminación, etc.; B15. Reducción del consumo de agua); y B2) una variable (B2. Hoteles que realizan inversiones medioambientales que repercuten directamente en el medio ambiente) representativas de inversiones medioambientales que repercuten directamente en el medio ambiente. Entre las primeras, se encuentran incluidos algunos de los 37 criterios de turismo sostenibles elaborados por la Organización Mundial del Turismo, concretamente, aquellos que incluyen en el apartado que la propia OMT califica como "Reducción de los impactos negativos con el medio ambiente".

En el segundo bloque se incluyen variables relativas a aspectos corporativos por contrastar:

a) Variables cualitativas: Aa. País de pertenencia (Bulgaria, Chipre, España, Francia, Grecia, Hungría, Polonia, Portugal, Reino Unido, Rumanía y Ucrania), Ab. Tipo de información financiera (consolidated data / unconsolidated data), Ac. Práctica contable (IFRS / Local GAAP).

b) Variables cuantitativas: Ba. Activo no corriente, Bc. Número de empleados, $B d$. ROI, Be. ROE, Bf. Leverage, Bg. Solvencia.

Las técnicas estadísticas aplicadas a los datos —usando el programa estadístico SPSS versión 15.0, tras recodificar las variables cualitativas, discretizar las cuantitativas a partir de los cuartiles y analizar las correlaciones existentes entre las variables el estudio mediante el coeficiente de correlación lineal de Pearson-fueron las siguientes:

1. El Cluster Analisys (método jerárquico aglomerativo de Ward) y método no jerárquico de las K-medias (Quick cluster) (Tarí et al., 2009) para intentar identificar distintos niveles de compromiso medioambiental entre los hoteles cotizados europeos.

2. La regresión logística para analizar la relación existente entre compromiso medioambiental y performance a través del contraste de la hipótesis $\mathrm{H}_{1}$.

$\mathrm{HO}_{\mathrm{I}}$ : La rentabilidad empresarial (ROI) no varía en función del nivel de compromiso medioambiental asumido. 
3. El análisis de la varianza (ANOVA) para determinar los factores que impulsan a las empresas a comprometerse con el medio ambiente mediante el contraste de la hipótesis $\mathrm{HO}_{2}$.

$\mathrm{HO}_{2}$ : El tamaño (activo no corriente, número de empleados), la ubicación geográfica dentro de la Unión, la práctica contable adoptada, la pertenencia a grupos, la rentabilidad económica y financiera (ROI y ROE) y la solvencia (Solvencia, Endeudamiento) de los hoteles cotizados europeos no varía en función del nivel de compromiso medioambiental asumido.

De forma generalizada, la literatura previa que pretende relacionar responsabilidad social con rentabilidad empresarial utiliza como técnica estadística el ANOVA (García Rodríguez y Armas Cruz, 2007; Tarí et al., 2009) y el análisis de regresión (Tarí et al., 2009). En este trabajo se utiliza como medida del performance la variable ROI (Return on Investment), tal como hacen García Rodríguez et al. (2006), dado que es un buen indicador del funcionamiento de una empresa en la medida en que representa su capacidad para remunerar al capital invertido, independientemente de su origen (fondos propios o préstamos), y que permite relativizar los diferentes tamaños empresariales.

\section{Resultados}

Niveles de compromiso medioambiental identificados entre los hoteles cotizados europeos de la población

Los resultados del análisis del cluster jerárquico realizado —utilizando el método aglomerativo de Ward - nos mostraron, a través del dendograma, la existencia de dos agrupaciones o comportamientos bien diferenciados entre la población de 69 hoteles cotizados europeos. Dentro de una de ellas, era posible observar, además, otras dos agrupaciones. Por ello, procedimos a realizar el análisis cluster no jerárquico K-medias con dos grupos y con tres grupos.

Tal como se muestra en el Anexo II, en el análisis del cluster realizado con dos grupos resultaron significativas $\mathrm{y}$, por tanto, diferenciadoras de las agrupaciones C2_1 y C2_2; todas las variables fueron utilizadas con un nivel de confianza del $100 \%$. En cuanto al análisis del cluster con tres grupos resultaron significativas y diferenciadoras de las agrupaciones C3_1, C3_2 y C3_3, también con un nivel de confianza del $100 \%$ las siguientes variables: dentro del grupo de variables re- 
presentativas del nivel de compromiso medioambiental asumido, las variables A3 (elaboración de un informe de evaluación del impacto ambiental), A4 (posesión de la certificación ISO 14000), A5 (posesión de otras certificaciones), A64, 66, 67, 68 (información, en memoria de sostenibilidad, de los criterios GRI relativos a los aspectos biodiversidad; emisiones, vertidos y residuos; productos y servicios; cumplimiento normativo; y nivel de aplicación, respectivamente); en cuanto a las variables representativas del tipo de actuación medioambiental emprendida, no resultó significativa la variable relativa a las inversiones medioambientales que repercuten directamente en el medio ambiente, pero sí lo fueron todas aquellas representativas de las actuaciones medioambientales que afectan al rendimiento económico del hotel, entre las que se encuentran B11 (política de compras favorecedora de productos ambientalmente amigables), B12 (uso más eficiente de las materias primas), B13 (adopción de medidas para reducir el consumo total de energía y fomento del uso de energía renovable), B14 (disminución de la contaminación del aire y del suelo y la causada por el ruido, la iluminación, etc.) y B15 (reducción del consumo de agua).

Por tanto, podemos concluir que entre los hoteles cotizados europeos registrados en AMADEUS, que son los que integran nuestra población, se distinguen las siguientes categorías o agrupaciones en función del compromiso medioambiental asumido:

1. Hoteles no comprometidos con el medio ambiente (C2_1 ó C3_1 = 61 hoteles). En esta agrupación se encuentran la mayoría de los hoteles cotizados pertenecientes a la Unión Europea, los cuales carecen de políticas o programas de protección del medio ambiente dado que su nivel de compromiso es nulo. Dentro de este grupo de hoteles llama especialmente la atención el caso de Orbis, S.A. - el mayor grupo turístico y hotelero en Polonia y en el centro de Europa-, pues a pesar de que publica en la web gran cantidad de información financiera (cuentas anuales, carta del director, informe de auditoría, etc.) no proporciona ningún dato acerca de compromiso alguno con el medioambiente. Lo mismo sucede con el Danubius Hotel and Spa (de Hungría), el C.C.C. Tourist Enterprises Public Company (de Chipre) y el Lampsa Hellenic Hotels (de Grecia). Se trata, no obstante, de casos aislados, puesto que en la sección Factores que influyen en el nivel de compromiso medioambiental adquirido por los hoteles cotizados europeos se demuestra la existencia de una relación directa y positiva entre tamaño y compromiso medioambiental en los hoteles analizados. 
2. Hoteles comprometidos con el medio ambiente (C2_2 = 8 hoteles). Esta agrupación integra un reducido grupo de hoteles cuyo nivel de compromiso con el medioambiente viene marcado, principalmente, por la posesión o no de un SGMA tipo EMAS (sistema comunitario de gestión y auditoría medioambientales) y por la repercusión del tipo de actuación medioambiental emprendida, ya sea que únicamente afecte al rendimiento económico del hotel o repercuta directamente en el medio ambiente. Así, dentro de esta categoría de hoteles es posible distinguir entre aquellos que poseen un nivel de compromiso medioambiental bajo y los que poseen un nivel de compromiso medioambiental elevado.

2.1 Hoteles con un nivel de compromiso bajo (C3_2 $=4$ hoteles $)$. Incluye hoteles que "afirman" estar comprometidos con el medio ambiente por poseer una política o un programa de gestión medioambiental. Sin embargo, los valores de los centros de los conglomerados finales que muestra el análisis del cluster en el Anexo II manifiestan, en relación con el nivel de compromiso medioambiental asumido, que ninguno de los cuatro hoteles de esta categoría se encuentra certificado con la ISO 14001 ni con cualquier otra certificación, ni posee un SGMA tipo EMAS. Además, la única actuación medioambiental emprendida por ellos, la cual afecta directamente a su rendimiento económico, consiste en la adopción de medidas para reducir el consumo total de energía y fomentar el uso de energías renovables. Ninguno de los hoteles de esta agrupación realiza inversiones medioambientales que repercutan directamente en el medio ambiente. Más bien se trata de un grupo de hoteles que divulga información sobre responsabilidad medioambiental con el propósito de legitimar sus actuaciones ante terceros, mas que con la finalidad de contribuir a la conservación del medioambiente, tal como sostiene la teoría de la legitimidad.

2.2 Hoteles con un nivel de compromiso elevado (C3_3 = 4 hoteles): Incluye cuatro hoteles cuyo mayor nivel de compromiso medioambiental manifiesta la posesión de un SGMA tipo EMAS y la realización de una serie de generosas actuaciones medioambientales que influyen tanto en el propio rendimiento económico del hotel como en el medioambiente directamente. Entre el primer tipo de actuaciones se encuentran la adopción de una política de compras verde, el uso eficiente de las materias primas, la asunción de medidas para reducir el consumo total de energía y el fomento del uso de la energía renovable, la disminución de la contaminación del aire y del 
suelo y la causada por el ruido, la iluminación, etc., así como la reducción del consumo de agua. En cuanto a las actuaciones medioambientales que repercuten directamente en el medio ambiente (variable B2), cabe citar la implementación por parte de Intercontinental Hotel Group de una serie de iniciativas ambientales que incluyen el reciclaje y mejora de la gestión de residuos y el uso de la energía eólica (aproximadamente siete millones de kilovatios-hora de electricidad que el InterContinental Willard de Washington DC consume todos los años produce cero $\mathrm{CO}_{2}$ ), o el caso del Hotel Verde del Grupo Hotelero Whitbread (Premier Inn in Tamworth) que reúne a las mejores tecnologías verdes disponibles en la actualidad para reducir el consumo energético hasta en un $80 \%$ y aumentar la eficiencia.

Sólo dos hoteles cotizados españoles que integran esta agrupación, Sol Meliá y NH Hoteles, están certificados con la ISO 14001; además, este último posee otras certificaciones como Green Key, Licencia Ambiental Única y Certificado Ambiental, y es el único hotel cotizado europeo de la población verificado externamente por la Global Reporting Initiative, organización que le otorgó el nivel de aplicación B+ por los criterios de las directrices G3 (tercera versión) cumplidos en su memoria de sostenibilidad 2007. Las memorias de sostenibilidad del resto de hoteles de este cluster, Sol Meliá, Intercontinental Hotel Group y Whitbread, están elaboradas a partir de los criterios GRI, aunque éstas no se encuentran verificadas por la organización.

\section{Relación existente entre compromiso medioambiental y rentabilidad empresarial}

Una vez identificadas y definidas las tres categorías de comportamiento entre la población de hoteles cotizados europeos en relación con el nivel de compromiso medioambiental asumido, se contrastó la hipótesis nula, $\mathrm{H}_{1}$, de igualdad de los valores medios de la variable rentabilidad económica (ROI) en los tres grupos:

$\mathrm{HO}_{\mathrm{I}}$ : La rentabilidad empresarial (ROI) no varía en función del nivel de compromiso medioambiental asumido.

En el cuadro 1 se presenta el resumen del modelo Logit propuesto para cada una de las dos regresiones realizadas; en él se observa que con un porcentaje de casos correctamente clasificados del $88.4 \%$ en ambos Logit resulta significativo a un nivel de confianza superior al 95\% de la variable Return on Investment (ROI). Además, 
el hecho de que esta variable haya resultado significativa en ambas categorías de hoteles y con un gran poder diferenciador en sentido opuesto, ratifica la bondad de las dos agrupaciones de hoteles.

\section{Cuadro 1}

\section{Resumen del modelo Logit propuesto}

\begin{tabular}{l|c|c|c|c|c|c}
\hline \multicolumn{3}{|c|}{ Variables dependientes } \\
\hline \multirow{2}{*}{$\begin{array}{l}\text { Variables } \\
\text { explicativas }\end{array}$} & \multicolumn{3}{|c|}{$\begin{array}{c}\text { C2_1 } \\
\text { (hoteles no comprometidos) }\end{array}$} & \multicolumn{3}{|c|}{$\begin{array}{c}\text { C2_2 } \\
\text { (hoteles comprometidos) }\end{array}$} \\
\cline { 2 - 7 } & $\beta$ & Sig. & $\operatorname{Exp}(\beta)$ & $\beta$ & Sig. & $\operatorname{Exp~(\beta )~}$ \\
\hline $\begin{array}{l}\text { Return on } \\
\text { investment } \\
\text { (ROI) }\end{array}$ & -1.181 & 0.018 & 0.307 & 1.181 & 0.018 & 3.258 \\
\hline & \multicolumn{3}{|c|}{$\%$ de clasificación: $88.4 \%$} & \multicolumn{3}{c}{$\%$ de clasificación: 88.4\% } \\
\hline
\end{tabular}

$\mathrm{C} 2 \_1=\beta 1(\mathrm{ROI}) ; \mathrm{C} 2 \_2=\beta 1(\mathrm{ROI})$

El cuadro 2 muestra el resultado del contraste estadístico Chi cuadrado para evaluar el ajuste global del modelo en cada una de las dos regresiones realizadas. El contraste arroja un buen valor predictivo del modelo puesto que éste resulta significativo al menos al 5\% (nivel de significación de 0.004). El cuadro 3, por su parte, indica que el modelo final clasifica correctamente en el $88.4 \%$ de los casos.

\section{Cuadro 2}

\section{Pruebas omnibus sobre los coeficientes del modelo}

\begin{tabular}{c|c|c|c|c}
\hline & & Chi cuadrado & gl & Sig. \\
\hline \multirow{3}{*}{ Paso 1 } & Paso & 8.163 & 1 & 0.004 \\
\cline { 2 - 5 } & Bloque & 8.163 & 1 & 0.004 \\
\cline { 2 - 5 } & Modelo & 8.163 & 1 & 0.004 \\
\hline
\end{tabular}




\section{Cuadro 3}

\section{Clasificación}

\begin{tabular}{|c|c|c|c|c|c|}
\hline & \multirow{3}{*}{\multicolumn{2}{|c|}{ Observado }} & \multicolumn{3}{|c|}{ Pronosticado } \\
\hline & & & \multicolumn{2}{|c|}{$\mathrm{C} 21$} & \multirow{2}{*}{$\begin{array}{l}\text { Porcentaje } \\
\text { correcto }\end{array}$} \\
\hline & & & 0.00 & 1.00 & \\
\hline \multirow{6}{*}{ Paso 1} & $\mathrm{C} 2 \_1$ & 0.00 & 0 & 8 & 0.0 \\
\hline & & 1.00 & 0 & 61 & 100.0 \\
\hline & \multicolumn{4}{|c|}{ Porcentaje global } & 88.4 \\
\hline & \multirow{3}{*}{\multicolumn{2}{|c|}{ Observado }} & \multicolumn{3}{|c|}{ Pronosticado } \\
\hline & & & \multicolumn{2}{|c|}{$\mathrm{C} 2 \_2$} & Porcentaje \\
\hline & & & 0.00 & 1.00 & correcto \\
\hline \multirow{3}{*}{ Paso 1} & \multirow[t]{2}{*}{$\mathrm{C} 2 \_2$} & 0.00 & 61 & 0 & 100.0 \\
\hline & & 1.00 & 8 & 0 & 0.0 \\
\hline & \multicolumn{4}{|c|}{ Porcentaje global } & 88.4 \\
\hline
\end{tabular}

El valor de corte es .500

A la vista de los resultados obtenidos es posible afirmar, en términos de probabilidades estimadas, que a un nivel de confianza del $95 \%$ existe una probabilidad del $88.4 \%$ de que un hotel, cuya rentabilidad económica sea elevada, esté más comprometido con el medio ambiente que otro que presente una rentabilidad económica menor. Se rechaza, por tanto, la hipótesis nula H0 planteada, según la cual la rentabilidad empresarial no varía en función del nivel de compromiso medioambiental asumido. Concretamente, el valor medio que alcanza el ROI en ambas agrupaciones es de 0.0870 y 0.005 para las categorías "Hoteles comprometidos" y "Hoteles no comprometidos", respectivamente. El elevado porcentaje de aciertos obtenidos garantiza que para el resto de empresas de la muestra y de la población, de cuyos datos no disponemos, los resultados serán similares.

Ésta es nuestra aportación al "argumento comercial" que nos planteamos como objetivo al inicio del trabajo: la existencia de una relación directa y positiva entre desempeño medioambiental y rentabilidad empresarial por parte de los hoteles cotizados europeos estudiados. Por tanto, con nuestro estudio respaldamos que la rentabilidad económica es una ventaja atribuible a la integración de las cuestiones medioambientales en la estrategia corporativa de los hoteles cotizados europeos de nuestra población.

Para finalizar, dado que para enriquecer cualquier análisis sobre el comportamiento medioambiental empresarial es preciso conocer los factores que empujan a las em- 
presas a dicho comportamiento, en la siguiente sección se analiza la influencia de estos factores en la población de hoteles estudiados.

Factores que influyen en el nivel de compromiso medioambiental adquirido por los hoteles cotizados europeos

Con el objeto de comprobar si el tamaño, el país de pertenencia, la práctica contable, la pertenencia a grupos, la rentabilidad y la solvencia son factores determinantes del nivel de compromiso medioambiental adquirido por los hoteles cotizados europeos, se contrastó, mediante el estadístico de contraste $\mathrm{F}$, la hipótesis nula $\mathrm{H}_{2}$ de igualdad de medias en los tres grupos de hoteles detectados con el análisis del cluster.

$\mathrm{HO}_{2}$ : El tamaño (activo no corriente, número de empleados), la ubicación geográfica dentro de la Unión, la práctica contable adoptada, la pertenencia a grupos, la rentabilidad económica y financiera (ROI y ROE) y la solvencia (Solvencia, Endeudamiento) de los hoteles cotizados europeos no varía en función del nivel de compromiso medioambiental asumido.

El cuadro 4 muestra las variables que resultaron significativas en el ANOVA y sus descriptivos (valores medios y frecuencias). Como puede observarse, en las tres agrupaciones resultan significativas las mismas variables, lo cual evidencia la existencia de diferencias entre las tres categorías de hoteles y añade consistencia al análisis del cluster realizado. Concretamente, a un nivel de confianza del 95\%, en las tres categorías de hoteles identificadas resultan significativas las variables de tamaño (medida mediante el número de empleados) y de práctica contable (IFRS -International Financial Reporting Standards- o Local Gaap). Las variables de rentabilidad (ROI — Return on Investment - y ROE — Return on Equity), de endeudamiento (Leverage) y de tamaño (activo no corriente) son significativas a un 95\% para las agrupaciones C3_1 (Hoteles no comprometidos) y C3_3 (Hoteles con compromiso elevado), pero para la agrupación C3_2 (Hoteles con compromiso bajo) sólo lo son a un $82 \%$ aproximadamente. Las únicas variables que no resultan significativas son la solvencia a corto plazo y el país de pertenencia; no obstante, las frecuencias de esta última variable muestran cómo los hoteles cotizados españoles e ingleses son los que presentan un mayor compromiso medioambiental concentrándose en el resto de países los hoteles que carecen de política de protección del medio ambiente. 
Cuadro 4

Resultados de los ANOVA

\begin{tabular}{|c|c|c|c|c|c|c|c|c|c|}
\hline \multirow[t]{2}{*}{ Variables } & \multicolumn{3}{|c|}{$\begin{array}{c}\text { Grupo } 1 \\
\text { No comprometidos }\end{array}$} & \multicolumn{3}{|c|}{$\begin{array}{c}\text { Grupo 2 } \\
\text { Compromiso bajo }\end{array}$} & \multicolumn{3}{|c|}{$\begin{array}{c}\text { Grupo 3 } \\
\text { Compromiso elevado }\end{array}$} \\
\hline & Sig & \multicolumn{2}{|c|}{ Media/Frec } & Sig & \multicolumn{2}{|c|}{ Media/Frec } & Sig & \multicolumn{2}{|c|}{ Media/Frec } \\
\hline Anc & 0.002 & \multicolumn{2}{|c|}{46996.19} & 0.173 & \multicolumn{2}{|c|}{944380.79} & 0.005 & \multicolumn{2}{|c|}{2542335.50} \\
\hline No. empleados & 0.000 & \multicolumn{2}{|l|}{266} & 0.063 & \multicolumn{2}{|l|}{5.367} & 0.005 & 17.743 & \\
\hline Consolidación & 0.000 & $\begin{array}{l}\text { Cons } \\
\text { Uncons } \\
\text { Total }\end{array}$ & $\begin{array}{l}9 \\
52 \\
61\end{array}$ & 0.11 & $\begin{array}{l}\text { Cons } \\
\text { Uncons } \\
\text { Total }\end{array}$ & $\begin{array}{l}3 \\
1 \\
4\end{array}$ & 0.000 & $\begin{array}{l}\text { Cons } \\
\text { Uncons } \\
\text { Total }\end{array}$ & $\begin{array}{l}4 \\
0 \\
4\end{array}$ \\
\hline País & 0.156 & $\begin{array}{l}\text { Bulgar } \\
\text { Cyprus } \\
\text { France } \\
\text { Greece } \\
\text { Hunga } \\
\text { Poland } \\
\text { Portug } \\
\text { Romani } \\
\text { Ukraine } \\
\text { UK } \\
\text { Total }\end{array}$ & $\begin{array}{l}1 \\
5 \\
5 \\
4 \\
1 \\
1 \\
1 \\
39 \\
1 \\
3 \\
61\end{array}$ & 0.720 & $\begin{array}{l}\text { France } \\
\text { UK } \\
\text { Total }\end{array}$ & $\begin{array}{l}1 \\
3 \\
4\end{array}$ & 0.114 & $\begin{array}{l}\text { Spain } \\
\text { UK } \\
\text { Total }\end{array}$ & $\begin{array}{l}2 \\
2 \\
4\end{array}$ \\
\hline $\begin{array}{l}\text { Práctica } \\
\text { contable }\end{array}$ & 0.000 & $\begin{array}{l}\text { IFRS } \\
\text { Local } \\
\text { Total }\end{array}$ & $\begin{array}{l}8 \\
53 \\
61 \\
\end{array}$ & 0.002 & $\begin{array}{l}\text { IFRS } \\
\text { Local } \\
\text { Total }\end{array}$ & $\begin{array}{l}3 \\
1 \\
4 \\
\end{array}$ & 0.000 & $\begin{array}{l}\text { IFRS } \\
\text { Local } \\
\text { Total }\end{array}$ & $\begin{array}{l}4 \\
0 \\
4 \\
\end{array}$ \\
\hline ROI & 0.007 & 0.0005 & & 0.173 & 0.0848 & & 0.021 & 0.0892 & \\
\hline ROE & 0.006 & -0.1067 & & 0.165 & 0.1155 & & 0.020 & 1.1450 & \\
\hline Leverage & 0.006 & 1.2376 & & 0.170 & 0.9243 & & 0.019 & 10.0211 & \\
\hline Solvencia & 0.327 & 6.6814 & & 0.989 & 1.5746 & & 0.173 & 0.6060 & \\
\hline
\end{tabular}

Con estos resultados podemos rechazar la hipótesis nula $\left(\mathrm{HO}_{2}\right)$ de igualdad de medias y afirmar, en términos estáticos, que existe una relación significativa y positiva entre el nivel de compromiso medioambiental asumido por los hoteles cotizados europeos y los factores tamaño - medido a través de las variables activo no corriente y número de empleados-, rentabilidad (económica y financiera), normativa contable adoptada por el hotel — bien sea la local o la internacional-y la comunicación de información contable no consolidada o consolidada.

A la vista de los valores medios y frecuencias que presentan estas variables significativas en el ANOVA (cuadro 4), podemos concluir que "los hoteles comprometidos con el medio ambiente" (grupo 3) se caracterizan por poseer mayor tamaño y mayor rentabilidad económica y financiera que el resto, presentar la información 
contable consolidada por pertenecen a grupos y elaborar dicha información según la normativa IFRS.

Además, en cuanto al nivel de endeudamiento (Leverage), los hoteles con un nivel de compromiso medioambiental elevado son los más apalancados $(\mathrm{L}=10.0211)$, lo cual podría representar riesgo para sus acreedores. Sin embargo, este apalancamiento financiero es positivo por el efecto que provoca la deuda en los valores del ROE (Rentabilidad de los fondos propios) debido al mayor valor que la rentabilidad empresarial (ROI) presenta sobre el costo financiero de la deuda. Los hoteles con un nivel de compromiso medio parecen financiar sus inversiones en mayor medida con fondos propios que con deuda, aunque el escaso nivel de apalancamiento financiero que manifiestan es también positivo. No obstante, los hoteles no comprometidos con el medio ambiente se encuentran más endeudados que los que adoptan un compromiso bajo al presentar un apalancamiento financiero negativo.

A pesar del escaso nivel de compromiso con el medio ambiente que presentan los hoteles europeos estudiados, el análisis de los factores determinantes de dicho nivel de compromiso nos ha permitido conocer el perfil del hotel comprometido. Se trata de un grupo hotelero de considerable tamaño que presenta sus cuentas anuales consolidadas según la normativa IFRS y cuyas rentabilidades, económica y financiera, son elevadas, al igual que su apalancamiento financiero que, además, es positivo.

\section{Conclusiones}

Este trabajo ha sido elaborado con el propósito de contribuir a la construcción de un marco de referencia objetivo para medir los avances en sostenibilidad de la actividad turística, cuya importancia se justifica en Garrod y Fyall (1998).

Para ello, se ha elaborado una propuesta de variables de medida del nivel de compromiso medioambiental, asumido por una muestra de hoteles cotizados europeos, a partir de los escasos trabajos existentes en la literatura previa y de los esfuerzos realizados por la Organización Mundial del Turismo; con lo anterior se ha pretendido contribuir, en la medida de lo posible, a la construcción del citado marco de referencia. Además, se ha evaluado el nivel de compromiso medioambiental asumido por los hoteles cotizados europeos a partir de los valores asignados a las variables propuestas mediante el análisis de los contenidos publicados en las web corporativas de los hoteles, advirtiéndose que la mayoría de los hoteles cotizados 
de la Unión Europea analizados carecen de políticas o programas de protección del medio ambiente. Cabe destacar que un reducido porcentaje de hoteles manifiesta un comportamiento que obedece a la tan contrastada teoría de la legitimidad: son hoteles que - a pesar de "afirmar" estar comprometidos con el medio ambienteen realidad no están certificados, ni elaboran memoria de sostenibilidad, y cuya política medioambiental se reduce a algunas prácticas de lo que Carmona Moreno et al. (2004) denominan compromiso medioambiental básico, como ahorro de agua y energía, recogida de residuos sólidos, etc; es decir, únicamente realizan actuaciones medioambientales que, según Blanco et al. (2009), afectan directamente a su rendimiento económico, además de que manifiestan una escasa concientización con las cuestiones medioambientales.

Por otro lado, dentro del reducidísimo grupo de hoteles estrechamente comprometidos con el medioambiente, es decir, aquellos que poseen un sistema de gestión medioambiental consolidado, tipo EMAS, se encuentran dos hoteles cotizados españoles (NH Hoteles y Sol Meliá) y dos del Reino Unido (Whitbread e Intercontinental Hotels Group). Todos ellos manifiestan un comportamiento medioambiental avanzado, pues adoptaron un enfoque de compromiso medioambiental a largo plazo. Además, no sólo realizan actuaciones que repercuten directamente en sus resultados, sino que llevan a cabo inversiones para mejorar directamente los resultados del medio ambiente y cumplen algunos de los 37 criterios de turismo sostenible de la OMT. Estos hallazgos coinciden con los resultados de las investigaciones llevadas a cabo por Pricewaterhouse Coopers en los catorce hoteles más grandes de Europa, los cuales revelan que nueve de los catorce hoteles analizados - entre los que se encuentran los cuatro hoteles mencionados anteriormente - tienen políticas de protección del medio ambiente.

Otro de los propósitos perseguidos en este trabajo ha sido la delimitación del perfil de hotel comprometido, dada la necesidad planteada por Husillos (2007) de profundizar en esta cuestión como complemento al análisis de la información sobre compromiso medioambiental publicada por las empresas para llegar a conclusiones fiables al respecto. Así, los hoteles más comprometidos con el medio ambiente son los que poseen mayor tamaño y mayor rentabilidad económica y financiera, los que presentan la información contable consolidada por pertenecer a grupos y los que elaboran dicha información según la normativa internacional.

Finalmente, con este trabajo también se ha pretendido contribuir al tan debatido "argumento comercial, pues entendemos que la consolidación de las actuaciones 
responsables con el medio ambiente, como un factor determinante de la rentabilidad empresarial, podría llegar a constituir un poderoso incentivo para las empresas. En este sentido, los resultados alcanzados demuestran que, para la muestra de hoteles estudiados, es posible compatibilizar el turismo responsable con el medioambiente y con la competitividad del negocio.

Para concluir, cabe resaltar que la diversidad de iniciativas voluntarias encontradas entre los hoteles de la población puede representar, tal como apunta García de Cortázar (2008), un obstáculo para las políticas europeas de RSE y desincentivar a las empresas para emprender acciones más creíbles y ambiciosas, aunque dado que los mercados y las empresas europeas se encuentran en diferentes fases de desarrollo, un modelo armonizado implicaría un rechazo significativo por parte de las empresas cuando no se respetase su idiosincrasia. En cualquier caso, ante el peligro de que la naturaleza voluntaria de estas actuaciones medioambientales pueda suponer una simple estrategia diseñada para legitimar las actividades corporativas ante los ojos de la comunidad (Lehman, 2004; Hopwood, 2009; Neu et al., 1998; Husillos Carqués, 2007; Hopwood, 2009), se hace necesario continuar realizando investigaciones, como la que aquí se expone, de cara a la construcción de un marco de referencia de medida, lo más objetivo posible, de los avances en sostenibilidad de la actividad turística.

\section{Referencias}

Adams, C.A. y G.R. Frost (2006). Accessibility and functionality of the corporate web site: implications for sustainability reporting. Business Strategy and the Environment 154: 275-287.

Aranguren, N. y E. Ochoa (2008). Divulgación de información sobre empleados y medioambiente en España y Alemania: una nota de investigación. Revista de Contabilidad 112: 123-142.

Archel Domench, P. y F. Lizarraga Dallo (2000). La divulgación de información medioambiental en España: evolución de la misma en el período 1995- 1998. Comunicación presentada en el IX Encuentro de Profesores Universitarios de Contabilidad, Las Palmas de Gran Canaria: 259-271. 
(2001). Algunos determinantes de la información medioambiental divulgada por las empresas españolas cotizadas. Revista de ContabilidadSpanish Accounting Review 447: 129-153.

Blanco, E., J. Rey-Maquieira y J. Lozano (2008). Economic incentives for tourism firms to undertake voluntary environmental management. Tourism Management 30: 112-122.

Beaumont, J., L. Pederson y B. Whitaker (1993). Managing the Environment. Oxford: Butterworth-Heinemann, citado en F. García Rodríguez e Y. Armas Cruz (2006). Relation between social-environmental responsibility and performance in hotel firms. Hospitality Management 26: 824-839.

Bien, A. (2006). Una guía simple para la certificación del turismo sostenible y el ecoturismo. Center for Ecotourism and Sustainable Development.

Blanco, E., J. Rey-Maquieira y J. Lozano (2008). Economic incentives for tourism firms to undertake voluntary environmental management. Tourism Management 30: 112-122.

Buhr, N. y M. Freedman (1996). A comparison of mandated and voluntary environmental disclosures. Critical perspectives on Accounting Conference, New York.

Carmona-Moreno, E., J. Céspedes-Lorente y J. Burgos-Giménez (2004). Environmental strategies in Spanish hotels: contextual factors and performance. The Service Industries Journal 24: 101-130.

Carrasco Fenech, F. y C. Larrinaga González (1995). Organizaciones, contabilidad y el entorno natural: Una perspectiva andaluza. Revista Española de Financiación y Contabilidad XXIV(83): 393-416.

Claver-Cortés, E., J.F. Molina-Azorín, J. Pereira-Moliner y M. D. López-Gamero, (2007). Environmental Strategies and Their Impact on Hotel Performance. Journal of Sustainable Tourism 15(6): 663-679. 
Chung, L. H. y L. D. Parker (2009). Managing social and environmental action and accountability in the hospitality industry: A Singapore perspective. Accounting Forum, doi: 10.1016/j.accfor.2009.10.003.

De Beelde, I. y E. Willequet (2000). External environmental reports: comparing practices in european countries. 23 Congreso Anual de la European Accounting Association, Munich, citado en A. Llull Gilet (2001). Contabilidad medioambiental y desarrollo sostenible en el sector turístico. Tesis Doctoral, Universitat de les Illes Balears. ISBN: 84-7632-877-X.

De Burgos, J. y J.J. Céspedes (2001). La protección ambiental y el resultado. Un análisis crítico de su relación. Investigaciones Europeas de Dirección y Economía de la Empresa 7(2): 93-108, citado en F. García Rodríguez e Y. Armas Cruz (2006). Relation between social-environmental responsibility and performance in hotel firms. Hospitality Management 26: 824-839.

Deegan, C. y M. Rankin (1996). Do australian companies report environmental news objectively? An analysis of environmental disclosures by firms prosecuted successfully by the Environemental Protection Authority. Accounting, Auditing \& Accountability Journal 9(2): 50-67.

Erdogan, N. y E. Baris (2006). Environmental protection programs and conservation practices of hotel in Ankara, Turkey. Tourism Management 28: 604614.

Fédération Des Experts Comptables Européens (1996). Research paper on expert statements in environmental reports. Bruselas: FEE.

García de Cortázar y Nebreda, C. (2008). Políticas comunitarias en RSE. Jornada políticas y prácticas de Responsabilidad Social Foro RSE.

García Rodríguez, F. e Y. Armas Cruz (2006). Relation between social-environmental responsibility and performance in hotel firms. Hospitality Management 26: 824-839.

Garrod, B. y A. Fyall (1998). Beyond the rhetoric of sustainable tourism? Tourism Management 193: 199-212. 
Guimaraes, T. y K. Liska (1995). Exploring the business benefits of Environmental stewardship. Business Strategy and the Environment 41: 9-22.

Giner Inchausti, B. (1992). La responsabilidad social de la empresa: la información medioambiental. Técnica Contable 527: 681-692.

Global Reporting Initiative 2000-2006. Guía para la elaboración de Memorias de Sostenibilidad, versión 3.0.

Hart, S. (1995). A natural resource-based view of the firm. Academy of Management Review 20 (4): 986-1014.

Hart, S. y G. Aguja (1996). Does it pay to be green? An empirical examination of the relationship between emission reduction and firm performance. Business Strategy and the Environment 5: 30-37.

Harte, G. y D. Owen (1991). Environmental disclosure in the annual reports of british companies: a research note. Accounting, Auditing and Accountability Journal 4 (3).

Hopwood, A. G. (2009). Accounting and the environment. Accounting, Organizations and Society 34: 433-439.

Husillos Carqués, F.J. (2007). An approach to the environmental disclosures of the listed Spanish firms form the legitimacy theory. Revista Española de Financiación y Contabilidad 133: 97-121.

Huybers, T. y F. Bennett (2002). Environmental management and the competitiveness of nature-based tourism destinations. Cheltenham: Edward Elgar Publishing.

Judge, W. y T. Douglas (1998). Performance implications of incorporating natural environmental issues into the strategic planning process: an empirical assessment. Journal of Management Studies 35: 241-262.

Kassinis, G. I. y A.C. Soteriou (2003). Greening the service profit chain: the impact of environmental management practices. Production and Operations Management 12: 368-402. 
Kippendorf, K. (1990). Metodología de análisis de contenido: teoría y práctica, Barcelona: Paidós, citado en F.J. Husillos Carqués. An approach to the environmental disclosures of the listed Spanish firms form the legitimacy theory. Revista Española de Financiación y Contabilidad 133: 97-121.

Klassen, R. y D. Whybark (1999). The impact of environmental technologies on manufacturing performance. Academy of Management Journal 42: 599-615.

Larriñaga, C., J.M. Moneva, F. Llena, F. Carrasco y C. Correa (2002). Regulación contable de la información medioambiental. Normativa española e internacional. Madrid: AECA.

, F. Llena, J.M. Moneva, F. Carrasco y C. Correa (2002). Accountability and accounting regulation: The case of the Spanish environmental disclosure standard. The European Accounting Review 11 (4): 723-740.

Lehman, Glen (2004). Social and environmental accounting. Trends and thoughts for the future. Accounting Forum, 28: 1-5, citado en A. Llull Gilet (2001). Contabilidad medioambiental y desarrollo sostenible en el sector turístico, tesis doctoral, Universitat de les Illes Balears. ISBN: 84-7632-877-X.

Llena, F. (1999). La contabilidad en la interacción empresa-medio ambiente. Su contribución a la gestión medioambiental, tesis doctoral, Universidad de Zaragoza. España.

Llena, F., J.M. Moneva, y V. Hernández (2007). Environmental Disclosures and Compulsory Accounting Standards: the case of Spanish annual reports. Business Strategy and the Environment XVI1: 50-63.

Llull Gilet, A. (2004). Los impactos medioambientales de la actividad turística. Revista interdisciplinar de gestión ambiental 6 (72):3-12.

McKercher, B. (1993). The unrecognized threat to tourism: can tourism survive sustainability? Tourism Manage 14 (2):131-136, citado en B. Garrod y A. Fyall (1998). Beyond the rhetoric of sustainable tourism? Tourism Management 19 (3): 199-212. 
Mensah, I. (2004). Environmental management practices in US hotels. Retrieved May, 2004, disponible en http://www.hotel-online.com/News/PR2004_2nd/ May04_Environmental practices.html.

Moneva Abadía, J. M. y F. Llena Maraculla (1996). Análisis de la información sobre responsabilidad social en las empresas industriales que cotizan en bolsa. Revista Española de Financiación y Contabilidad 87: 361-401.

Moneva, J.M., P. Archel y C. Correa (2006). GRI and the camouflaging of corporate unsustainability. Accounting Forum 302: 121-137.

Neu, D., H. Warsame y K. Pedwell (1998). Managing public impressions: environmental disclosures in annual reports. Accounting, Organizations and Society 233: 265-282.

Niskala, M. y M. Pretes (1995). Environemntal reporting in Finland: a note on use of annual reports. Accounting, Organizations and Society 17: 595-612.

Parra, E., F. J. García y D. Gutiérrez (2004). Importance of environmental resuources and capabilities for firm's competitiveness. The case of the hotel industry in the Canary Islands. Anatolia 15: 183-149.

Peyró Vilaplana, E. (1997). Información social y ambiental en España: Un estudio empírico. IX Congreso de AECA, Salamanca: 681-703.

Porter, M.E. e Y.C. Vander Linde (1995). Green and competitive: ending the stalemate. Harvard Business Review 735: 120-134.

PriceWaterhouse Coopers (2006). Corporate Responsibility in the hospitality sector: pain or gain? Hospitality Directions. Europe Edition 13, marzo.

Reglamento sustituye al Reglamento (CEE) no 1836/93 del Consejo, de 29 de junio de 1993, por el que se permite que las empresas del sector industrial se adhieran con carácter voluntario a un sistema comunitario de gestión y auditoría medioambientales. 
Reglamento (CE) n ${ }^{\circ}$ 761/2001 del Parlamento Europeo y del Consejo, de 19 de marzo de 2001, por el que se permite que las organizaciones se adhieran con carácter voluntario a un sistema comunitario de gestión y auditoría medioambientales (EMAS).

Rivera, J. (2004). Institutional pressures and voluntary environmental behavior in developing countries: evidence from the Costa Rican hotel industry. Society and Natural Resources 17: 779-797.

Ripoll Feliu, V. M. y C. Crespo Soler (1998). Costes derivados de la gestión medioambiental. Técnica Contable 591.

Roberts, C.B. (1991). Environmental disclosures: a note on reporting practices in mainland Europe. Accounting, Auditing and Accountability Journal 4(3).

Russo, M.V. y P.A. Fouts (1997). A resource-based perspective on corporate environmental performance and profitability. Academy of Management Journal 40 (3): 534-559.

Shrivastava, P. (1995). Environmental technologies and competitive advantage. Strategic Management Journal 163: 183-200.

Tarí, J.J., E. Claver-Cortés, J. Pereira-Moliner y J.F. Molina-Azorín (2009). Levels of quality and environmental management in the hotel industry: Their joint influence on firm performance. International Journal of Hospitality Management 29 (3): 500-510.

Thomas, A. (2001). Corporate environmental policy and abnormal stock price returns: an empirical investigation. Business Strategy and the Environment 10 (3): $125-134$.

Trung, D. N. y S. Kumar (2005). Resource use and waste management in Vietnam hotel industry. Journal of Cleaner Production 134: 109-116.

Venkatraman, N. y V. Ramanujam (1986). Measures of business performance in strategy research: A comparison of approaches. Academy of Managament Review 11: 801-814. 
Wagner, M. et al. (2002). The relationship between the environmental and economic performance of firms: an empirical analysis of the European paper industry. Corporate Social Responsibility and Environmental Management 9: 133-146, citado en J.J. Tarí, E. Claver-Cortés, J. Pereira-Moliner y J.F. Molina-Azorín (2009). Levels of quality and environmental management in the hotel industry: Their joint influence on firm performance. International Journal of Hospitality Management 29 (3): 500-510.

Worrell, D., K.M. Gilley, W.D. Davison III y A. El-Jely (1995). When green turns to red: stock market reaction to announced greening activities. Paper Presented at the academy of management meeting, Vancouver. 


\section{Anexo 1 \\ Población objeto de estudio, ordenada de mayor a menor tamaño (en función del nivel medio de activos)}

\begin{tabular}{|c|c|c|}
\hline Nombre de la compañía & País & Bolsa \\
\hline WHITBREAD PLC & UNITED KINGDOM & $\begin{array}{l}\text { London Stock Exchange } \\
\text { (SETS) }\end{array}$ \\
\hline NH HOTELES S.A. & SPAIN & Mercado Continuo Español \\
\hline $\begin{array}{l}\text { MILLENNIUM \& COPTHORNE } \\
\text { HOTELS PLC }\end{array}$ & UNITED KINGDOM & $\begin{array}{l}\text { London Stock Exchange } \\
\text { (SETS) }\end{array}$ \\
\hline SOL MELIA S.A. & SPAIN & Mercado Continuo Español \\
\hline $\begin{array}{l}\text { INTERCONTINENTAL HOTELS } \\
\text { GROUP PLC }\end{array}$ & UNITED KINGDOM & $\begin{array}{l}\text { London Stock Exchange } \\
\text { (SETS) }\end{array}$ \\
\hline MWB GROUP HOLDINGS PLC & UNITED KINGDOM & $\begin{array}{l}\text { London Stock Exchange } \\
\text { (SETS) }\end{array}$ \\
\hline ORBIS S.A. & POLAND & Warsaw Stock Exchange \\
\hline $\begin{array}{l}\text { DANUBIUS HOTEL AND SPA } \\
\text { PUBLIC LIMITED COMAPNY }\end{array}$ & HUNGARY & Budapest Stock Exchange \\
\hline IONIAN HOTEL ENTERPRISES S.A. & GREECE & Athens Stock Exchange \\
\hline ASTIR PALACE VOULIAGMENI S.A. & GREECE & Athens Stock Exchange \\
\hline $\begin{array}{l}\text { LEPTOS CALYPSO HOTELS PUBLIC } \\
\text { LIMITED }\end{array}$ & CYPRUS & Cyprus Stock Exchange \\
\hline $\begin{array}{l}\text { C.C.C. TOURIST ENTERPRISES } \\
\text { PUBLIC COMPANY LIMITED }\end{array}$ & CYPRUS & Cyprus Stock Exchange \\
\hline $\begin{array}{l}\text { LAMPSA HELLENIC HOTELS CO. } \\
\text { S.A. }\end{array}$ & GREECE & Athens Stock Exchange \\
\hline $\begin{array}{l}\text { CONSTANTINOU BROS HOTELS } \\
\text { PUBLIC COMPANY LIMITED }\end{array}$ & CYPRUS & Cyprus Stock Exchange \\
\hline AMATHUS PUBLIC LIMITED & CYPRUS & Cyprus Stock Exchange \\
\hline $\begin{array}{l}\text { TURISM,HOTELURI,RESTAURANTE } \\
\text { MAREA NEAGRA SA }\end{array}$ & ROMANIA & Bucharest Stock Exchange \\
\hline BUCURESTI TURISM SA & ROMANIA & RASDAQ \\
\hline $\begin{array}{l}\text { IMOBILIARIA CONSTRUTORA } \\
\text { GRAO PARA, S.A. }\end{array}$ & PORTUGAL & Euronext Lisbon \\
\hline LES HOTELS DE PARIS & FRANCE & Euronext Paris \\
\hline ASTARTI DEVELOPMENT PLC & CYPRUS & Cyprus Stock Exchange \\
\hline $\begin{array}{l}\text { COMPANIA HOTELIERA } \\
\text { INTERCONTINENTAL ROMANIA SA }\end{array}$ & ROMANIA & RASDAQ \\
\hline GEKE S.A. & GREECE & Athens Stock Exchange \\
\hline LONRHO PLC & UNITED KINGDOM & $\begin{array}{l}\text { London Stock Exchange } \\
\text { (SEAQ) }\end{array}$ \\
\hline GOLDEN SANDS AD & BULGARIA & Bulgarian Stock Exchange \\
\hline PEEL HOTELS PLC & UNITED KINGDOM & $\begin{array}{l}\text { London Stock Exchange } \\
\text { (SEAQ) }\end{array}$ \\
\hline
\end{tabular}




\begin{tabular}{|c|c|c|}
\hline $\begin{array}{l}\text { BIROUL DE TURISM PENTRU } \\
\text { TINERET (B.T.T.) SA }\end{array}$ & ROMANIA & RASDAQ \\
\hline BEGA TURISM SA & ROMANIA & RASDAQ \\
\hline $\begin{array}{l}\text { SOC IMMOBILIERE ET EXPLOIT } \\
\text { HOTEL MAJES }\end{array}$ & FRANCE & Euronext Paris \\
\hline HOTELIM & FRANCE & Euronext Paris \\
\hline HOTEL REGINA PARIS & FRANCE & Euronext Paris \\
\hline BALNEOCLIMATERICA SA & ROMANIA & RASDAQ \\
\hline MAYPOLE GROUP PLC & UNITED KINGDOM & $\begin{array}{l}\text { London Stock Exchange } \\
\text { (SEAQ) }\end{array}$ \\
\hline $\begin{array}{l}\text { CATERING INTERNATIONAL ET } \\
\text { SERVICES (C.I.S.) }\end{array}$ & FRANCE & Euronext Paris \\
\hline PERLA MAJESTIC SA & ROMANIA & RASDAQ \\
\hline COCOR TURISM SA & ROMANIA & RASDAQ \\
\hline INTERNATIONAL SA & ROMANIA & RASDAQ \\
\hline Nombre de la compañía & País & Bolsa \\
\hline PALACE SA & ROMANIA & RASDAQ \\
\hline MOLDOVA ESTIVAL 2002 SA & ROMANIA & RASDAQ \\
\hline DORNA TURISM SA & ROMANIA & RASDAQ \\
\hline $\begin{array}{l}\text { DE TURISM,HOTELURI SI } \\
\text { RESTAURANTE PRAHOVA SA }\end{array}$ & ROMANIA & RASDAQ \\
\hline $\begin{array}{l}\text { STE HOTELIERE ET IMMOBILIERE } \\
\text { DE NICE }\end{array}$ & FRANCE & Euronext Paris \\
\hline $\begin{array}{l}\text { SOCIETATEA COMERCIALA DE } \\
\text { TRATAMENT BALNEAR BUZIAS SA }\end{array}$ & ROMANIA & RASDAQ \\
\hline $\begin{array}{l}\text { CHEREMOSH TURYSTYCHNYY } \\
\text { KOMPLEKS }\end{array}$ & UKRAINE & PFTS Stock Exchange \\
\hline HERCULES SA & ROMANIA & RASDAQ \\
\hline NEPTUN-OLIMP SA & ROMANIA & RASDAQ \\
\hline $\begin{array}{l}\text { HYDRO HOTEL EASTBOURNE } \\
\text { PUBLIC LIMITED COMPANY }\end{array}$ & UNITED KINGDOM & Plus Markets \\
\hline TERRA ESTIVAL 2002 SA & ROMANIA & RASDAQ \\
\hline HOTEL CLUB ESTIVAL 2002 SA & ROMANIA & RASDAQ \\
\hline GRANAT SA & ROMANIA & RASDAQ \\
\hline JIUL SA CRAIOVA & ROMANIA & RASDAQ \\
\hline OLIMP ESTIVAL 2002 SA & ROMANIA & RASDAQ \\
\hline $\begin{array}{l}\text { IMPARATUL ROMANILOR TURISM } \\
\text { SA }\end{array}$ & ROMANIA & RASDAQ \\
\hline ROMANTA ESTIVAL 2002 SA & ROMANIA & RASDAQ \\
\hline CAPITOL SA & ROMANIA & RASDAQ \\
\hline RUSCA SA HUNEDOARA & ROMANIA & RASDAQ \\
\hline
\end{tabular}




\begin{tabular}{l|l|l}
\hline DURAU SA & ROMANIA & RASDAQ \\
\hline TRANSILVANIA ESTIVAL 2002 SA & ROMANIA & RASDAQ \\
\hline TURISM SI AGREMENT “OLTUL” SA & ROMANIA & RASDAQ \\
\hline BANAT ESTIVAL 2002 SA & ROMANIA & RASDAQ \\
\hline TREI BRAZI SA & ROMANIA & RASDAQ \\
\hline CLABUCET ESTIVAL 2002 SA & ROMANIA & RASDAQ \\
\hline COMPLEX HOTELIER PARC SA & ROMANIA & RASDAQ \\
\hline AMARA SA & ROMANIA & RASDAQ \\
\hline BORCEA SA & ROMANIA & RASDAQ \\
\hline TURIST SEMENIC SA & ROMANIA & RASDAQ \\
\hline MIORITA 2001 SA & ROMANIA & RASDAQ \\
\hline PARINGUL SA & ROMANIA & RASDAQ \\
\hline ATHENEE PALACE SA & ROMANIA & RASDAQ \\
\hline APOLLO SA & ROMANIA & RASDAQ \\
\hline
\end{tabular}

Fuente: Base de datos AMADEUS

\section{Anexo 2}

\section{Resultados de análisis cluster con 2 y 3 agrupaciones}

a) Cluster con 2 agrupaciones

\section{Número de casos en cada conglomerado}

\begin{tabular}{ccc|c}
\cline { 2 - 3 } Conglomerado & 1 & 61.000 \\
\cline { 2 - 3 } & 2 & 8.000 \\
Válidos & & 69.000 \\
Perdidos & .000 \\
\hline
\end{tabular}




\begin{tabular}{|c|c|c|c|c|c|c|}
\hline \multicolumn{7}{|c|}{ ANOVA } \\
\hline & \multicolumn{2}{|c|}{ Conglomerado } & \multicolumn{2}{|l|}{ Error } & \multirow{2}{*}{$\begin{array}{l}\text { F } \\
\text { Media } \\
\text { cuadrática }\end{array}$} & \multirow{2}{*}{$\begin{array}{l}\text { Sig. } \\
\text { gl }\end{array}$} \\
\hline & $\begin{array}{l}\text { Media } \\
\text { cuadrática }\end{array}$ & GI & $\begin{array}{l}\text { Media } \\
\text { cuadrática }\end{array}$ & gl & & \\
\hline A1_NO COMPR & 7.072 & 1 & .000 & 67 & . & . \\
\hline A2_PROG MA & 7.072 & 1 & .000 & 67 & . & . \\
\hline A3_INF EVALUAC & 24.864 & 1 & .013 & 67 & 1903.882 & .000 \\
\hline A4_ISO 14000 & 21.659 & 1 & .022 & 67 & 967.454 & .000 \\
\hline A5_OTRAS CERTIF & 21.659 & 1 & .022 & 67 & 967.454 & .000 \\
\hline A6_EMAS & 15.913 & 1 & .030 & 67 & 533.087 & .000 \\
\hline A61_GRI MATER & 1.768 & 1 & .030 & 67 & 59.232 & .000 \\
\hline A62_GRI ENERG & 1.768 & 1 & .030 & 67 & 59.232 & .000 \\
\hline A63_GRI AGUA & 1.768 & 1 & .030 & 67 & 59.232 & .000 \\
\hline A64_GRI BIODIV & 2.763 & 1 & .058 & 67 & 47.768 & .000 \\
\hline A65_GRI EMISIO & 1.768 & 1 & .030 & 67 & 59.232 & .000 \\
\hline A66_PROD_SS & 2.763 & 1 & .058 & 67 & 47.768 & .000 \\
\hline $\begin{array}{l}\text { A67_CUMPL } \\
\text { NORM }\end{array}$ & 2.763 & 1 & .058 & 67 & 47.768 & .000 \\
\hline $\begin{array}{l}\text { A68_NIVEL } \\
\text { APLICACIÓN }\end{array}$ & .111 & 1 & .013 & 67 & 8.462 & .005 \\
\hline B11_COMP VERDE & 13.371 & 1 & .028 & 67 & 477.804 & .000 \\
\hline B12_USO MP & 11.051 & 1 & .022 & 67 & 493.599 & .000 \\
\hline B13_CONS ENERG & 8.951 & 1 & .013 & 67 & 685.398 & .000 \\
\hline B14_CONTAMIN & 11.051 & 1 & .022 & 67 & 493.599 & .000 \\
\hline B15_CONS AGUA & 11.051 & 1 & .022 & 67 & 493.599 & .000 \\
\hline B2_INV MA & 15.913 & 1 & .030 & 67 & 533.087 & .000 \\
\hline
\end{tabular}

Las pruebas F sólo se deben utilizar con una finalidad descriptiva, puesto que los conglomerados han sido elegidos para maximizar las diferencias entre los casos en diferentes conglomerados. Los niveles críticos no son corregidos, por lo que no pueden interpretarse como pruebas de la hipótesis de que los centros de los conglomerados son iguales. 


\section{Centros de los conglomerados finales}

\begin{tabular}{l|l|l}
\cline { 2 - 3 } & \multicolumn{2}{c}{ Conglomerado } \\
\cline { 2 - 3 } & $\mathbf{1}$ & $\mathbf{2}$ \\
\hline A1_NO COMPR & 1 & 2 \\
\hline A2_PROG MA & 2 & 1 \\
\hline A3_INF EVALUAC & 0 & 2 \\
\hline A4_ISO 14000 & 0 & 2 \\
\hline A5_OTRAS CERTIF & 0 & 2 \\
\hline A6_EMAS & 0 & 2 \\
\hline A61_GRI MATER & 0 & 1 \\
\hline A62_GRI ENERG & 0 & 1 \\
\hline A63_GRI AGUA & 0 & 1 \\
\hline A64_GRI BIODIV & 0 & 1 \\
\hline A65_GRI EMISIO & 0 & 1 \\
\hline A66_PROD_SS & 0 & 1 \\
\hline A67_CUMPL NORM & 0 & 1 \\
\hline A68_NIVEL APLICACIÓN & 0 & 0 \\
\hline B11_COMP VERDE & 0 & 1 \\
\hline B12_USO MP & 0 & 1 \\
\hline B13_CONS ENERG & 0 & 1 \\
\hline B14_CONTAMIN & 0 & 1 \\
\hline B15_CONS AGUA & 0 & 1 \\
\hline B2_INV MA & 0 & 2 \\
\hline
\end{tabular}

b) Cluster con 3 agrupaciones

Número de casos en cada conglomerado

\begin{tabular}{c|c|c}
\hline \multirow{2}{*}{ Conglomerado } & 1 & 61.000 \\
\cline { 2 - 3 } & 2 & 4.000 \\
\cline { 2 - 3 } & 3 & 4.000 \\
\hline Válidos & 69.000 \\
\hline Perdidos & .000 \\
\hline
\end{tabular}




\begin{tabular}{|c|c|c|c|c|c|c|}
\hline & \multicolumn{5}{|c|}{ ANOVA } & \multirow{3}{*}{$\begin{array}{c} \\
\text { Sig. } \\
\text { gl }\end{array}$} \\
\hline & \multicolumn{2}{|c|}{ Conglomerado } & \multicolumn{2}{|l|}{ Error } & \multirow{2}{*}{$\begin{array}{c}\text { F } \\
\text { Media } \\
\text { cuadrática }\end{array}$} & \\
\hline & $\begin{array}{c}\text { Media } \\
\text { cuadrática }\end{array}$ & gl & $\begin{array}{c}\text { Media } \\
\text { cuadrática }\end{array}$ & gl & & \\
\hline A1_NO COMPR & 3.536 & 2 & .000 & 66 & . & . \\
\hline A2_PROG MA & 3.536 & 2 & .000 & 66 & . & . \\
\hline A3_INF EVALUAC & 12.495 & 2 & .011 & 66 & 1099.522 & .000 \\
\hline A4_ISO 14000 & 11.080 & 2 & .015 & 66 & 731.261 & .000 \\
\hline A5_OTRAS CERTIF & 11.080 & 2 & .015 & 66 & 731.261 & .000 \\
\hline A6_EMAS & 8.957 & 2 & .000 & 66 & . & . \\
\hline A61_GRI MATER & 1.884 & 2 & .000 & 66 & . & . \\
\hline A62_GRI ENERG & 1.884 & 2 & .000 & 66 & . & . \\
\hline A63_GRI AGUA & 1.884 & 2 & .000 & 66 & . & . \\
\hline A64_GRI BIODIV & 2.944 & 2 & .011 & 66 & 259.058 & .000 \\
\hline A65_GRI EMISIO & 1.884 & 2 & .000 & 66 & . & . \\
\hline A66_PROD_SS & 2.944 & 2 & .011 & 66 & 259.058 & .000 \\
\hline $\begin{array}{l}\text { A67_CUMPL } \\
\text { NORM }\end{array}$ & 2.944 & 2 & .011 & 66 & 259.058 & .000 \\
\hline $\begin{array}{l}\text { A68_NIVEL } \\
\text { APLICACIÓN }\end{array}$ & .118 & 2 & .011 & 66 & 10.362 & .000 \\
\hline B11_COMP VERDE & 6.748 & 2 & .027 & 66 & 254.503 & .000 \\
\hline B12_USO MP & 5.775 & 2 & .015 & 66 & 381.174 & .000 \\
\hline B13_CONS ENERG & 4.538 & 2 & .011 & 66 & 399.348 & .000 \\
\hline B14_CONTAMIN & 5.775 & 2 & .015 & 66 & 381.174 & .000 \\
\hline B15_CONS AGUA & 5.775 & 2 & .015 & 66 & 381.174 & .000 \\
\hline B2_INV MA & 8.957 & 2 & .000 & 66 & . & . \\
\hline
\end{tabular}

Las pruebas F sólo se deben utilizar con una finalidad descriptiva, puesto que los conglomerados han sido elegidos para maximizar las diferencias entre los casos en diferentes conglomerados. Los niveles críticos no son corregidos, por lo que no pueden interpretarse como pruebas de la hipótesis de que los centros de los conglomerados son iguales. 


\section{Centros de los conglomerados finales}

\begin{tabular}{l|l|l|l}
\cline { 2 - 4 } & \multicolumn{3}{|c}{ Conglomerado } \\
\hline A1_NO COMPR & $\mathbf{1}$ & $\mathbf{2}$ & $\mathbf{3}$ \\
\hline A2_PROG MA & 1 & 2 & 2 \\
\hline A3_INF EVALUAC & 2 & 1 & 1 \\
\hline A4_ISO 14000 & 0 & 2 & 2 \\
\hline A5_OTRAS CERTIF & 0 & 2 & 2 \\
\hline A6_EMAS & 0 & 2 & 2 \\
\hline A61_GRI MATER & 0 & 2 & 1 \\
\hline A62_GRI ENERG & 0 & 0 & 1 \\
\hline A63_GRI AGUA & 0 & 0 & 1 \\
\hline A64_GRI BIODIV & 0 & 0 & 1 \\
\hline A65_GRI EMISIO & 0 & 0 & 1 \\
\hline A66_PROD_SS & 0 & 0 & 1 \\
\hline A67_CUMPL NORM & 0 & 0 & 1 \\
\hline A68_NIVEL APLICACIÓN & 0 & 0 & 1 \\
\hline B11_COMP VERDE & 0 & 0 & 0 \\
\hline B12_USO MP & 0 & 2 & 1 \\
\hline B13_CONS ENERG & 0 & 2 & 1 \\
\hline B14_CONTAMIN & 0 & 1 & 1 \\
\hline B15_CONS AGUA & 0 & 2 & 1 \\
\hline B2_INV MA & & 2 & 1 \\
\hline
\end{tabular}


